\title{
CRÉDITO RURAL E IMPACTO SOBRE O VALOR DA PRODUÇÃO AGROPECUÁRIA: UMA ANÁLISE PARA AGRICULTORES NÃO FAMILIARES
}

RURAL CREDIT AND IMPACT ON THE VALUE OF AGRICULTURAL PRODUCTION: AN ANALYSIS FOR NON-FAMILY FARMERS

Gabriela dos Santos Eusébio ${ }^{1}$

ORCID: https://orcid.org/0000-0001-9876-2609

\section{Alexandre Gori Maia²}

ORCID: https://orcid.org/0000-0003-0075-5094

\section{Rodrigo Lanna Franco da Silveira ${ }^{3}$}

ORCID: https://orcid.org/0000-0002-1208-5713

(Núcleo de Economia Agrícola e do Meio Ambiente (NEA) da Universidade Estadual de Campinas - Unicamp - Brasil) ${ }^{1,2,3}$

\section{RESUMO}

O objetivo deste trabalho foi analisar o impacto do acesso ao crédito rural no desempenho econômico dos estabelecimentos agropecuários não familiares no Brasil. Comparou-se, assim, o valor da produção agrícola dos produtores não familiares que acessaram ou não crédito rural, controlando as características do agricultor e do negócio. As análises se basearam nos microdados do Censo Agropecuário de 2006 do IBGE, com um total de 796.422 estabelecimentos. Para considerar as relações de dupla causalidade entre crédito e produção agropecuária, aplicou-se o método em dois estágios. Verificou-se que a obtenção de capital de terceiros impactou positivamente a produção agropecuária. Houve ainda evidências de que a intensidade de tal influência variou conforme a fonte de crédito obtido e entre as diferentes regiões brasileiras.

Palavras-chave: Crédito rural. Produção agropecuária. Produtores não familiares.

\section{ABSTRACT}

The purpose of this study was to analyze the impact of credit on agricultural production value of nonfamily farmers in Brazil. The study compared nonfamily farmers' production value considering the obtainment of credit, controlling for producers and farms characteristics. The data set consists of the 2006 Agricultural Census with 796,422 farmers. In order to take into account bicausal relationship between credit and agricultural production, the empirical analysis is conducted using two-stage method. Results suggested that credit access had a positive and significant impact on agricultural production value. There was also evidence that the intensity of such influence varied according to the source of credit obtained and across Brazilian regions.

Keywords: Agricultural credit. Agricultural production. Non-familiar farmers. 


\section{INTRODUÇÃO}

A análise da relação entre desenvolvimento do sistema financeiro e crescimento da economia tem sido objeto de estudo na literatura econômica desde os trabalhos seminais de Schumpeter (1911) e Robinson (1952), com análises posteriores de Gurley e Shaw (1955) e Goldsmith (1969). Uma das vertentes dessa investigação tem base na visão de "supply-leading", na qual as instituições financeiras, ao disponibilizarem crédito aos agentes econômicos, fornecem estímulo à inovação, o que, por sua vez, impulsiona a dinâmica da economia. Assim sendo, a transferência de recursos dos agentes superavitários aos deficitários acaba por aperfeiçoar a alocação de recursos e, assim, aumentar a produtividade marginal do capital (MATOS, 2002).

Ao incluir nesta análise o setor agrícola, é possível observar que os mercados financeiros impactam positivamente a atividade ao: i) ofertar produtos capazes de garantir gerenciamento dos riscos de preço, de crédito e de produção; ii) gerar liquidez na economia, disponibilizando recursos de forma a garantir um melhor planejamento e execução do negócio - incluem-se, neste contexto, crédito para custeio, investimento e comercialização da atividade; iii) estimular pesquisa e desenvolvimento com vistas à inovação tecnológica; iv) colaborar na geração de renda e redução das desigualdades no meio rural; v) promover benefícios para o produtor, permitindo, por exemplo, a regularização do seu fluxo de consumo pessoal, por meio da compatibilização de seu fluxo de renda contínuo ou sazonal; entre outros.
O financiamento agropecuário, no entanto, esbarra em dificuldades advindas das características do setor, que o torna mais arriscado do ponto de vista dos emprestadores. Yaron et al. (1997) e Spolador (2001) ressaltam que o alcance ao meio rural pelo sistema financeiro é dificultado por fatores como a renda rural, que tende a ser menor e mais volátil em comparação à renda urbana. Além disso, as operações financeiras neste segmento, em geral, possuem baixa escala com ausência de colateral. E, por fim, os mercados são fragmentados e isolados, o que amplia o problema de informação assimétrica entre credores e mutuários. Ao tentar minimizar tal assimetria, o sistema financeiro utiliza mecanismos de seleção e monitoramento dos tomadores, resultando em contratos complexos e exigências de garantias, as quais acabam por elevar os custos de transação (Stiglitz e Weiss, 1981). Esses fatores têm significativo impacto nas decisões de concessão de crédito e na avaliação dos riscos, aumentando o custo relacionado aos empréstimos de pequena magnitude, principalmente aos requeridos pelos pequenos produtores rurais. Devido a essas características, os financiamentos ao setor rural brasileiro são, majoritariamente, advindos de bancos oficiais, com encargos financeiros fixados pelo governo em níveis inferiores aos praticados no mercado, apesar do crescimento da participação das instituições privadas.

Grande parte dos estudos recentes nessa temática do crédito, tem dedicado atenção à avaliação de impactos do microcrédito (ROOYEN et al., 2012; KABOSKl; TOWNSEND, 2012; TU et al., 2015). No Brasil, foco especial tem sido conferido às análises de desempenho do Pronaf (Programa Nacional de Fortalecimento da Agricultura 
Familiar) (GUANZIROLI, 2007; DAMASCENO et al., 2011). No entanto, pouca atenção tem sido dada aos efeitos diferenciados do crédito sobre os pequenos e grandes estabelecimentos, inexistindo investigações baseadas em microdados no Brasil que avaliam tal questão, especificamente para a agricultura empresarial.

O presente trabalho analisa os determinantes do acesso ao crédito e seus impactos no desempenho econômico dos estabelecimentos agropecuários não familiares no Brasil. Utilizam-se os microdados do Censo Agropecuário de 2006, do Instituto Brasileiro de Geografia e Estatísticas (IBGE), que totalizam 796.422 agricultores não familiares. Pelo fato de não incluir agricultores familiares, os quais são caracterizados pela dedicação majoritária à autossubsistência', o estudo cobre uma lacuna na literatura sobre crédito ao direcionar a pesquisa aos produtores rurais empresariais. Embora estes representassem somente 16\% dos estabelecimentos em 2006, concentravam cerca de $70 \%$ da área total e $68 \%$ do valor total da produção agropecuária brasileira. A hipótese é que o acesso ao financiamento tem impacto positivo na produção agropecuária, colaborando para a geração de renda no meio rural.

\section{REVISÃO DE LITERATURA}

A análise do impacto do crédito rural sobre a atividade agropecuária se concentra, em geral, em países em desenvolvimento, onde tal setor tem significativa importância na economia. Diversos trabalhos se propõem a estimar os efeitos das políticas de crédito, seja sobre o crescimento do setor, sobre a produtividade das propriedades ou até mesmo sobre o rendimento das famílias residentes no meio rural.

O estudo de Binswanger e Khandker (1995) avaliou, por exemplo, o impacto da expansão do crédito rural em 85 distritos da Índia entre 1972 e 1981. Com uso de um modelo de equações simultâneas, constatou-se que a expansão dos financiamentos teve maior influência sobre emprego e produção não agrícola. No setor agrícola, verificou-se que o crédito ampliou o uso de fertilizantes, elevou o investimento privado em máquinas e na pecuária e, por fim, impactou positivamente os salários rurais. Sidhu et al. (2008) também investigaram o efeito do crédito na Índia. Com base em dados de 160 agricultores e do censo agropecuário de 1995 e 2001, os resultados da estimação de um modelo de equações simultâneas indicaram uma influência positiva do crédito no crescimento do setor agrícola, uma vez que o acesso ao capital possibilitou a adoção de insumos de produção mais modernos e investimentos privados em mecanização, irrigação, entre outros. Narayanan (2016), por sua vez, analisou a relação entre o crédito formal e o Produto Interno Bruto (PIB) agropecuário na Índia. Obteve-se que o crédito formal teve impactos nas compras de insumos e na mecanização. Porém, dado o contexto de baixas produtividade e eficiência técnica da atividade, o efeito do crédito no produto agropecuário se mostrou relativamente pequeno.

Pesquisas realizadas em outros países fornecem ainda relevantes informações a respeito da

\footnotetext{
1 São considerados como estabelecimentos de agricultura familiar aqueles dirigidos por um membro da família, com área total inferior a um limite regional, força de trabalho predominantemente familiar e renda proveniente do próprio estabelecimento.
} 
influência do crédito na área rural. Akram et al. (2013), por exemplo, estimaram, a partir de uma amostra de 152 agricultores paquistaneses, que a eficiência técnica média dos agricultores com acesso ao crédito foi superior em relação aos demais produtores. Para Khandker e Faruqee (2003), tanto o crédito formal como o informal são de grande importância para a agricultura, uma vez que capitaliza os agricultores e os estimulam a investir em novas tecnologias. Com base em dados do Agricultural Development Bank of Pakistan (ADBP) - instituição responsável por 90\% do crédito formal nas áreas rurais paquistanesas - e fazendo-se uso de um método de estimação em dois estágios, os autores mostraram que o crédito influenciou positivamente a prosperidade dos produtores, especialmente no grupo de pequenos proprietários. Já sob foco nas famílias rurais da China, Zhu e Li (2007) estimaram o impacto do crédito em toda a distribuição de riqueza desses agentes. Utilizando um modelo de regressão quantílica e dados agregados de 3000 famílias rurais, demonstrouse que a influência do crédito foi positiva para agricultores com rendimentos médios e baixos. Ainda com foco na China, Xin e Li (2011) avaliaram o efeito do crédito no desenvolvimento econômico agrícola na província de Heilongjiang. Ao aplicarem técnicas de séries temporais, os autores observaram uma relação positiva e estatisticamente significativa entre as variáveis. Por fim, Moura (2016) estudou a causalidade entre crédito rural e crescimento da atividade agropecuária no Brasil, constatando presença de causalidade unidirecional do crédito para o crescimento do produto agropecuário, inexistindo relação reversa.
Outro conjunto recente de estudos explorou os efeitos da restrição do crédito no setor agropecuário. Com base no mercado chinês, Dong et al. (2012) apontaram que a produtividade agrícola aumentou em $31,6 \%$ e o rendimento em $23,2 \%$, quando as barreiras ao financiamento foram eliminadas. Li et al. (2013) também pesquisaram sobre tal tema, observando que $61,5 \%$ das famílias rurais chinesas encontravam restrições ao crédito no período do estudo, o que levou a uma perda de 15,7\% do lucro líquido e a uma diminuição de 18,2\% nas despesas com consumo. Em estudo comparativo entre a Índia e a China, Kumar et al. (2013) demonstraram que, em 74\% (78\%) das 741 (400) famílias chinesas (indianas) pesquisadas nos anos de 2008 e 2009, a restrição de crédito resultou em uma diminuição de insumos utilizados na produção, levando à perda de produtividade.

Os países africanos também foram alvos de análise neste tema. Ali et al. (2014) verificaram que a eliminação do racionamento do crédito em Ruanda aumentava a produção agropecuária em aproximadamente 17\%. Já Foltz (2014) concluiu que o racionamento afetou a rentabilidade das propriedades rurais na Tunísia, levando a uma produção e alocação sub ótima de fatores de produção, como a terra, mão-de-obra e outros insumos. Para o Brasil, Lopes et al. (2011), a partir de 1720 estabelecimentos e utilizando mínimos quadrados ponderados e variáveis instrumentais, mostraram que os produtores que apresentavam melhor acesso ao mercado financeiro estariam em melhor situação, uma vez que a principal restrição ao crescimento da produção agrícola brasileira era o capital, com destaque ao capital de giro. 


\section{DADOS}

\subsection{CARACTERÍSTICAS DOS PRODUTORES E DOS ESTABELECIMENTOS}

As análises do efeito do acesso ao financiamento sobre o valor total da produção (VTP) basearamse em informações do Censo Agropecuário. A partir da variável "Agricultura familiar- lei 11326 de 24/07/2006", foram selecionados estabelecimentos que não se enquadravam neste conjunto de produtores, sendo definidos dois grupos. O primeiro, denominado "Grupo 1", foi composto pelos estabelecimentos sem acesso aos financiamentos em 2006 e o segundo, "Grupo 2", foi formado por aqueles que acessaram qualquer tipo de crédito - bancário e de outras fontes, tais como de fornecedores, parentes, cooperativas de crédito, financeiras, empresas integradoras, entre outros. Foram, então, analisadas as características desses agentes, de suas propriedades e do sistema de produção.

A variável de interesse principal das análises foi o logaritmo do VTP. A Tabela 1 apresenta os valores médios de tal variável, além das características socioeconômicas dos 796.422 agricultores não familiares em 20061. O Sul concentrou o maior número de agricultores com acesso ao financiamento (Grupo 2), com 49.067 observações, seguido pelo Sudeste, com 37.171 agricultores. Com exceção do Sul, onde 31,7\% dos estabelecimentos acessaram crédito em 2006, o percentual de acesso foi baixo nas demais regiões e menor naquelas menos desenvolvidas: $9,8 \%$ no
Norte, $11,4 \%$ no Nordeste, $16,8 \%$ no Sudeste, $15,6 \%$ no Centro-Oeste. No Norte e Nordeste, por exemplo, o número de estabelecimentos sem crédito foi aproximadamente nove e oito vezes maior do que os que conseguiram crédito, respectivamente.

Apesar do número menor de estabelecimentos com acesso a financiamento, o seu valor médio da produção foi superior em relação aos demais em todas as regiões. O Centro-Oeste apresentou o maior valor médio da produção, de cerca de $\mathrm{R} \$ 630$ mil para os estabelecimentos com acesso a crédito (Grupo 2), frente a $\mathrm{R} \$ 187$ mil do grupo sem acesso (Grupo 1). Ao analisar a produtividade média ( $R \$ /$ ha), a superioridade dos produtores com acesso ao financiamento se torna mais evidente, em todas as regiões, com destaque novamente para o CentroOeste.

A participação em cooperativas foi mais elevada entre os produtores com acesso ao financiamento, em todas as regiões, principalmente no Sul e no Sudeste (participação de 57,7\% e 45,8\% dos estabelecimentos, respectivamente). Os dados de escolaridade dos produtores que acessaram o crédito mostram que o Centro-Oeste e o Sudeste apresentavam maior percentual de indivíduos com ensino médio completo ou superior. O percentual de produtores que terminaram o ensino fundamental, ou alcançaram níveis superiores, foi mais elevado no grupo que acessou empréstimos, em todas as regiões, com exceção do Nordeste.

2 O Apêndice A apresenta as variáveis relativas às características do produtor utilizadas no trabalho. 


\subsection{CARACTERÍSTICAS DO SISTEMA DE PRODUÇÃO}

A Tabela 2 apresenta os valores médios das características do sistema de produção dos agricultores não familiares ${ }^{3}$. Uma aproximação para o grau de tecnificação de tais produtores é dada pela informação sobre o uso de tração. As regiões apresentaram percentuais de uso de tração animal muito próximos para os estabelecimentos que se utilizaram de empréstimos. Porém, quando se analisa os dados para tração mecânica, é possível perceber uma discrepância entre as diferentes regiões. Considerando o grupo com acesso ao crédito (Grupo 2), enquanto que, no Norte e Nordeste, o uso da tração mecânica não alcançou $40 \%$ dos estabelecimentos, no Centro-Sul do País, tal percentual foi superior a 75\% da amostra.

Quanto à orientação técnica e adoção de técnicas produtivas, o Grupo 2 apresentou melhores condições de produção. O acesso à orientação técnica também foi discrepante entre as regiões - levando em conta o Grupo 2, 77,7\% $(25,1 \%)$ dos agricultores não familiares do Sul (Nordeste) tiveram orientação técnica. Tal diferença também foi observada no percentual de realização de tratamentos no solo. Enquanto que, no Sul, 92,1\% dos agricultores não familiares com crédito realizaram algum tratamento no solo; no Norte, esse percentual foi de 29,4\%. Vale, contudo, salientar que, independentemente das diferenças regionais, os produtores com acesso ao crédito tiveram maior acesso à orientação técnica, realizaram tratamentos de solo com maior intensidade e contaram com maior percentual de tração mecânica.
Em relação à variável grau de especialização, esta é medida pela razão entre o valor da produção do produto agrícola principal e o valor total de produção. Sua análise foi realizada por quatro categorias: i) altamente especializado (ESE), com grau de especialização igual a 1; ii) especializada (ESP), com grau de especialização inferiores a 1 e superior a 0,65; iii) diversificada (EDIV), com grau de especialização entre 0,65 e 0,35; iv) muito diversificada (EMDIV), com grau de especialização menor do que 0,35. Em todas as regiões, o percentual de estabelecimentos especializados foi superior entre os estabelecimentos que acessaram crédito em 2006, com destaque ao Centro-Oeste que apresentou cerca de $80 \%$ do Grupo 2 com atividades altamente especializadas (variáveis ESE e ESP).

Por fim, a variável grau de integração do mercado é obtida pela razão entre a receita total da atividade agrícola e VTP, utilizando três categorias: i) altamente integradas (EMI), com grau de integração superior a 0,9; ii) integrada (EI), com grau de integração entre 0,5 e 0,9; iii) pouco integrados (EPI), com grau de integração entre zero e 0,5. O percentual de estabelecimentos integrados ou muito integrados ao mercado foi mais elevado entre o Grupo 2 (com acesso ao crédito), em todas as regiões, com exceção do Nordeste. O percentual se mostrou novamente elevado no Centro-Oeste, onde aproximadamente, $70 \%$ dos agricultores não familiares com acesso ao crédito estavam muito integrados ao mercado.

\footnotetext{
3 O Apêndice B apresenta as variáveis de sistema de produção utilizadas no trabalho.
} 


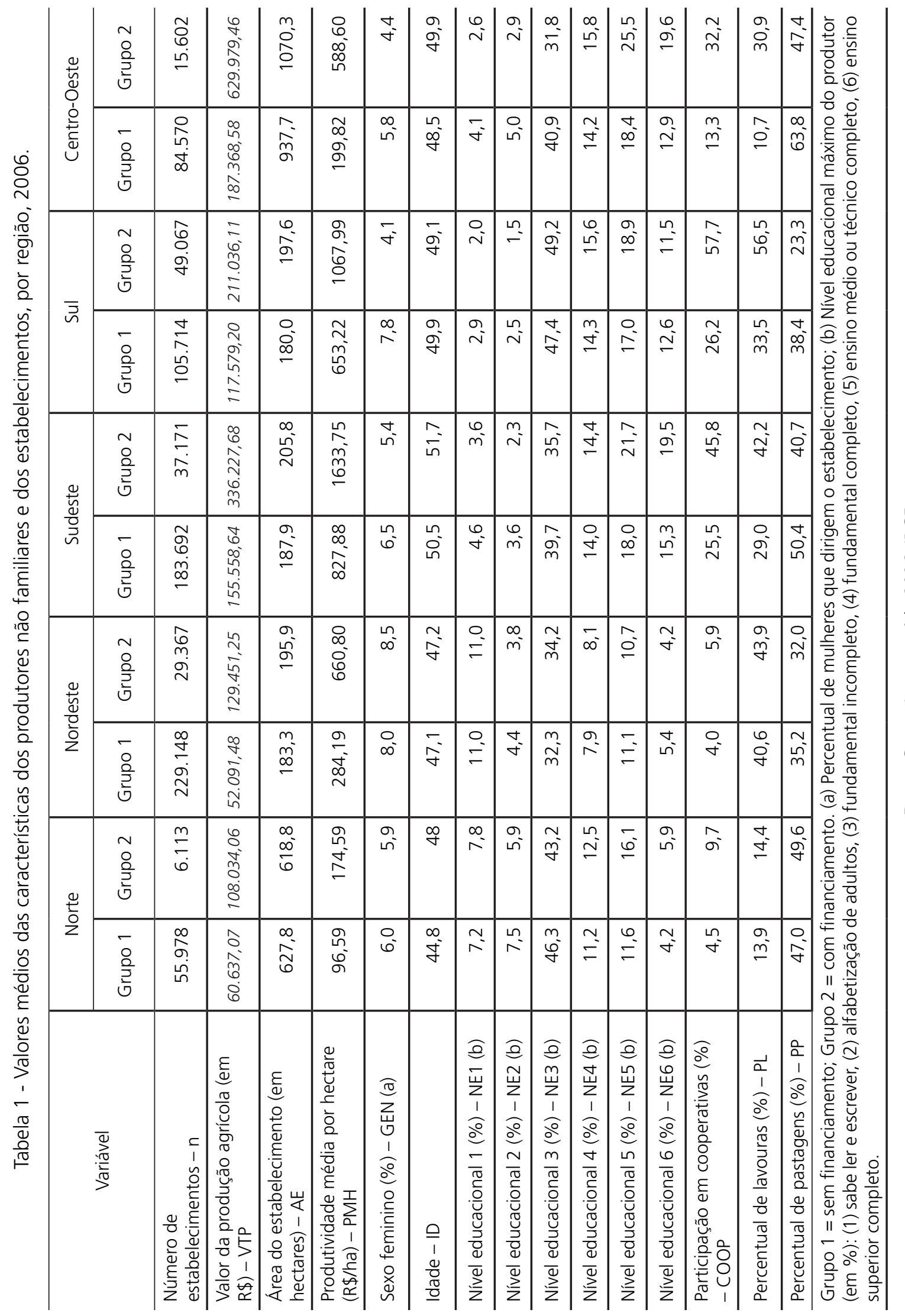


Tabela 2 - Valores médios das características do sistema de produção dos agricultores não familiares, por região, 2006.

\begin{tabular}{|c|c|c|c|c|c|c|c|c|c|c|}
\hline \multirow[b]{2}{*}{ Variável } & \multicolumn{2}{|c|}{ Norte } & \multicolumn{2}{|c|}{ Nordeste } & \multicolumn{2}{|c|}{ Sudeste } & \multicolumn{2}{|c|}{ Sul } & \multicolumn{2}{|c|}{ Centro-Oeste } \\
\hline & $\underset{1}{\text { Grupo }}$ & $\underset{2}{\text { Grupo }}$ & $\begin{array}{l}\text { Grupo } \\
1\end{array}$ & $\underset{2}{\text { Grupo }}$ & $\begin{array}{l}\text { Grupo } \\
1\end{array}$ & $\underset{2}{\text { Grupo }}$ & $\underset{1}{\text { Grupo }}$ & $\underset{2}{\text { Grupo }}$ & $\underset{1}{\text { Grupo }}$ & $\begin{array}{c}\text { Grupo } \\
2\end{array}$ \\
\hline $\begin{array}{l}\text { Tração animal e/ou mecânica (\%) - } \\
\text { TRAM }\end{array}$ & 49,3 & 64,9 & 59,2 & 68,4 & 71,2 & 87,4 & 71,7 & 94,7 & 76,9 & 91,8 \\
\hline Tração animal (\%) - TRA & 37,0 & 45,6 & 42,4 & 47,5 & 38,5 & 37,0 & 30,9 & 27,6 & 52,4 & 45,1 \\
\hline Tração mecânica (\%) - TRM & 22,3 & 36,1 & 30,3 & 38,8 & 52,0 & 75,8 & 55,6 & 85,8 & 53,2 & 79,9 \\
\hline Orientação técnica (\%) - OT & 25,3 & 41,7 & 17,9 & 25,1 & 46,6 & 67,7 & 47,0 & 77,7 & 42,9 & 69,2 \\
\hline Tratamento de solo (\%) - TS & 15,9 & 29,4 & 27,8 & 35,2 & 59,9 & 83,1 & 62,2 & 92,1 & 37,0 & 67,2 \\
\hline Agrotóxico (\%) - AGT & 17,8 & 34,3 & 24,7 & 39,5 & 33,2 & 61,3 & 45,8 & 83,9 & 21,7 & 52,5 \\
\hline $\begin{array}{l}\text { Estabelecimento superespecializado } \\
(\%)-\text { ESSE }\end{array}$ & 26,7 & 19,6 & 22,0 & 13,8 & 32,4 & 25,9 & 22,7 & 11,5 & 30,7 & 21,2 \\
\hline $\begin{array}{l}\text { Estabelecimento especializado (\%) } \\
\text { - ESP }\end{array}$ & 42,4 & 55,4 & 37,6 & 41,3 & 39,1 & 50,0 & 40,0 & 45,4 & 46,0 & 57,8 \\
\hline $\begin{array}{l}\text { Estabelecimento diversificado (\%) } \\
\text { - EDIV }\end{array}$ & 16,0 & 18,1 & 28,4 & 36,8 & 14,7 & 19,7 & 25,0 & 38,7 & 10,0 & 17,2 \\
\hline $\begin{array}{l}\text { Estabelecimento muito diversificado } \\
(\%) \text { - EMDIV }\end{array}$ & 1,9 & 1,9 & 3,6 & 5,7 & 1,2 & 1,4 & 2,8 & 3,2 & 0,5 & 0,5 \\
\hline $\begin{array}{l}\text { Estabelecimento muito integrado } \\
(\%)-\text { EMI }\end{array}$ & 36,2 & 46,0 & 32,2 & 29,1 & 49,0 & 61,2 & 41,1 & 57,0 & 51,3 & 69,7 \\
\hline Estabelecimento integrado (\%) - El & 22,9 & 27,6 & 22,7 & 29,8 & 17,1 & 21,0 & 22,0 & 27,3 & 17,1 & 16,9 \\
\hline $\begin{array}{l}\text { Estabelecimento pouco integrado } \\
(\%)-E P I\end{array}$ & 27,8 & 21,3 & 36,8 & 38,8 & 21,3 & 14,7 & 27,3 & 14,5 & 18,8 & 10,2 \\
\hline Número de trabalhadores - NT & 6,0 & 5,8 & 5,5 & 9,4 & 9,9 & 20,7 & 4,4 & 8,6 & 6,5 & 18,4 \\
\hline
\end{tabular}

Fonte: Censo Agropecuário 2006, IBGE.

\section{METODOLOGIA}

Para considerar a relação de dupla causalidade entre VTP e acesso ao crédito, utilizou-se o método de estimação em Dois Estágios (2E). O modelo com variável dependente escalar (YTP $=$ logVTP) foi, inicialmente, ajustado por Mínimos Quadrados Ordinários. Já os modelos com variáveis dependentes nominais ( $Y^{C_{-} \text {Banco }}$ e $Y^{\text {cr_outros}}$ ), sendo o não acesso a crédito a categoria de referência, foram ajustados por funções logísticas acumuladas utilizando o método de máxima verossimilhança.
Duas observações devem ser feitas em relação à estimação do modelo. Em primeiro lugar, optouse por diferenciar o grupo de produtores com acesso ao crédito (Grupo 2) segundo a fonte do financiamento obtido - de bancos (Cr_Bancos) ou por outras vias (Cr_Outros), incluindo cooperativas de crédito, fornecedores, comerciantes, empresas integradoras, outras financeiras, ONGs, parentes e outros agentes. Forneceu-se, assim, um melhor detalhamento do impacto do crédito sobre o desempenho do VTP nosestabelecimentos conforme as fontes do empréstimo. A segunda observação consiste no controle da heterogeneidade regional, realizado com uso de efeitos fixos para as 558 
microrregiões do país. As equações em sua forma estrutural foram:

$$
\begin{aligned}
& \int Y_{i}^{V T P}=\lambda_{0}+\lambda_{1} Y_{i}^{E}{ }^{\text {Banco }}+\lambda_{2} Y_{i}^{E}{ }_{-}{ }^{\text {Ourros }}+\sum \lambda_{j} X_{j}^{V T P}+u_{i} \\
& \left\{\mathrm{~h}\left(\frac{Y_{i}^{\epsilon}{ }_{-}^{\text {Banco }}}{1-Y_{i}^{\epsilon}{ }_{-B a c o}}\right)=\gamma_{0}+\gamma_{1} Y_{i}^{V T P}+\sum \gamma_{1 k} X_{k}^{\epsilon}+v_{1 i}\right. \\
& \mathrm{h}\left(\frac{Y_{i}^{E_{-} \text {Ooutros }}}{1-Y_{i}^{E_{-} \text {Outros }}}\right)=\gamma_{0}+\gamma_{2} Y_{i}^{V T P}+\sum \gamma_{2 k} X_{k}^{\epsilon}+v_{2 i}
\end{aligned}
$$

Em que $X_{j i}^{\text {VTP }}$ e $X_{k i}{ }^{c r}$ são os fatores exógenos que influenciam, respectivamente, O VTP e o acesso ao crédito do estabelecimento; ui e vi são erros aleatórios não explicados pelo modelo.

A existência de relação mútua entre VTP e acesso ao crédito exigiu estimativas em dois estágios para eliminar a inconsistência devida à existência de correlação entre variáveis independentes endógenas e os termos de erro (PINDYCK; RUBINFELD, 2004). No primeiro estágio, obtiveram-se variáveis instrumentais fortemente relacionadas às endógenas independentes, mas não correlacionadas aos termos de erro. Essas variáveis foram previstas por um sistema de equações reduzidas, no qual cada variável endógena foi ajustada em função de todas as variáveis exógenas do problema. Fez-se, assim, necessária a escolha de pelo menos uma variável relacionada ao financiamento, mas que não guardasse relação com o VTP. A variável escolhida foi o montante de dívidas do estabelecimento $(D T)$, uma vez que essa variável demonstra estreita relação com a decisão de emprestar ou não, por parte dos agentes fornecedores de crédito, mas não afeta diretamente a produção do estabelecimento e também não traz relação com a capacidade individual do produtor (Xl; LI, 2007). De maneira análoga, foram necessárias variáveis relacionadas ao VTP que não tivessem relação com o financiamento. Essas variáveis foram: tração animal (TRA), tração mecânica (TRM), tratamento do solo (TS) e uso de agrotóxico (AGT), as quais apresentam estreita relação com a produção do estabelecimento, mas não influenciam a decisão do agente de crédito em emprestar. As equações da forma reduzida são dadas em (2).

$$
\begin{aligned}
& Y_{i}^{V T P}=\pi_{0}+\sum \pi_{1 j} X_{j}^{V T P}+\sum \pi_{\mathrm{z} k} X_{k}^{\epsilon}+u_{1_{i}}^{\prime} \\
& \left\{\mathrm{h}\left(\frac{Y_{i}^{E}{ }_{-}^{\text {Banco }}}{1-Y_{i}^{E}{ }_{-}^{\text {Banco }}}\right)=\pi_{0}+\sum \pi_{1_{j}} X_{j}^{V T P}+\sum \pi_{2 k} X_{k}^{\epsilon}+u_{2_{i}}^{\prime}\right.
\end{aligned}
$$

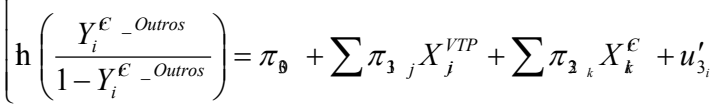

A ausência de variáveis independentes endógenas no sistema (2) garantiria, segundo os pressupostos de um modelo clássico de regressão linear, estimativas consistentes e não tendenciosas dos parâmetros das equações (PINDYCK; RUBINFELD, 2004). Posteriormente, no segundo estágio da análise, as variáveis independentes endógenas das equações estruturais, equação (1), foram substituídas pelos respectivos valores previstos na equação (2) - $\hat{Y}_{i}^{V T P}, \hat{Y}_{i}^{\epsilon}{ }^{B a n c o}$ e $\hat{Y}_{i}^{\epsilon}$ _outros O novo sistema de equações com variáveis instrumentais é dado por:

$$
\begin{aligned}
& Y_{i}^{V T P}=\lambda_{0}+\lambda_{1} \hat{Y}_{i}^{E}{ }_{-}^{\text {Banco }}+\lambda_{2} \hat{Y}_{i}^{E}{ }_{-}^{\text {Outros }}+\sum \lambda_{j} X_{j}^{V T P}+u_{i} \\
& \left\{\mathrm{~h}\left(\frac{Y_{i}^{\epsilon}{ }^{\text {Banco }}}{1-Y_{i}^{\epsilon}{ }^{\text {Banco }}}\right)=\gamma_{0}+\gamma_{1} \hat{Y}_{i}^{V T P}+\sum \gamma_{1 k} X_{k}^{\epsilon}+v_{1 i}\right. \\
& \mathrm{h}\left(\frac{Y_{i}^{\epsilon}{ }^{E} \text { Outros }}{1-Y_{i}^{\epsilon}{ }_{-} \text {Outros }}\right)=\gamma_{0}+\gamma_{2} \hat{Y}_{i}^{V T P}+\sum \gamma_{2 k} X_{k}^{\epsilon}+v_{2 i}
\end{aligned}
$$

As variáveis dependentes dos modelos para o acesso ao crédito - $\ln \left[Y_{i} /\left(1-Y_{i}\right)\right]$ - referem-se aos logaritmos naturais das respectivas chances de sucesso, ou seja, das razões entre as probabilidades de sucesso $(Y=1)$ sobre o fracasso $(Y=0)$. Essa razão 
expressa quantas vezes a chance de obter crédito é maior que a chance de não acessar empréstimo.

Por fim, vale observar que a existência de endogeneidade entre o crédito e o valor total da produção exigiu que a estimação do modelo fosse realizada em dois estágios. Para comparar os estimadores obtidos em um estágio (1E) com os estimadores em dois estágios (2E), utilizou-se o teste de especificação de Hausman. Sejam as equações, na forma estrutural, apresentadas em (1), o teste de especificação de Hausman avalia se

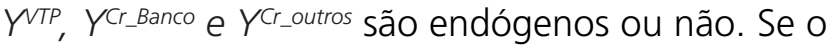
teste for significativo, as estimativas em um estágio e em dois estágios são distintas, sendo apenas as estimativas de dois estágios consistentes.

\section{RESULTADOS}

\subsection{DETERMINANTES DO ACESSO AO CRÉDITO}

As estimativas do modelo de regressão logística multinomial, que explicitam os determinantes do acesso ao crédito, são apresentadas no Apêndice C. Como mencionado na metodologia de pesquisa, analisa-se o crédito para cada tipo de fonte. Os empréstimos bancários foram predominantes no País em 2006 - enquanto que 15,2\% dos agricultores não familiares fizeram uso de tal fonte de capital, cerca de $2 \%$ dos estabelecimentos se utilizaram de outras vias de financiamento. Vale ressaltar que $82,7 \%$ dos agricultores não familiares não acessaram crédito.

Embora não seja objetivo central do trabalho, as estimativas dos coeficientes permitem obter importantes interpretações sobre os determinantes da obtenção de empréstimos. Os resultados mostram que o VTP foi um importante determinante do acesso ao crédito, sobretudo para aqueles que o acessaram de outras fontes além de bancos. Por outro lado, a obtenção de financiamento esteve inversamente relacionada ao tamanho do estabelecimento $(A E)$ e ao uso da mão de obra (NT). Esses resultados sugerem que o acesso ao crédito foi maior entre os estabelecimentos mais produtivos: com maior VTP e menor área e uso de mão de obra. Em relação às diferenças regionais, a análise demonstra que os estabelecimentos do Sudeste (SE) e, sobretudo, do Sul (SU) estiveram mais propensos a obter crédito de outras fontes. Os estabelecimentos do Sul foram também aqueles com as maiores chances de obter financiamento bancário. Como esperado, a participação em cooperativas $(C O O P)$ e entidades de classe $(E C)$ aumentou em grande medida a chance de obter empréstimo.

\subsection{DETERMINANTES DO VALOR BRUTO DA PRODUÇÃO}

A Tabela 3 mostra as estimativas do modelo de regressão. O ajuste se baseou em um conjunto de 699.501 observações com informações válidas; 96.921 observações foram desconsideradas por apresentarem valores nulos para pelo menos uma das variáveis em análise. A estimação por $2 \mathrm{E}$ se ajustou bem às informações da amostra, como demonstram as estatísticas de qualidade do ajuste.

As estimativas dos coeficientes associados ao acesso ao crédito foram substancialmente distintas entre os modelos de $1 \mathrm{E}$ e $2 \mathrm{E}$, sugerindo uma potencial fonte de viés nas estimativas de 1E com 
tendência de subestimar o impacto do crédito sobre a produção. A estimação do teste de especificação de Hausman ${ }^{4}$ se mostrou significativo para as variáveis de acesso ao crédito e para a variável de valor total da produção. Com isso, as estimativas de $1 \mathrm{E}$ e 2E são distintas, e apenas as estimativas de $2 \mathrm{E}$ serão consideradas. Dado o elevado número de observações da amostra, as estimativas de $2 \mathrm{E}$ podem ser consideradas consistentes.

Controlando as características dos estabelecimentos, houve diferença significativa no valor total da produção para agricultores não familiares com acesso ao crédito em relação aos demais. O coeficiente relativo à variável instrumental, $\hat{Y}_{i}^{\epsilon}{ }^{\epsilon}$ Banco, mostra que a obtenção de financiamento via banco elevou o valor médio total da produção em 63,3\% (e,49055-1). No caso de possuir financiamento por outros meios (cooperativas de crédito, fornecedores, comerciantes, empresas integradoras, outras financeiras, ONGs, parentes e outros agentes), o impacto no valor médio da produção total foi ainda mais elevado, 213\% superior $\left(e^{1,1421}-1\right)$.
Quanto aos coeficientes associados às características dos produtores, o modelo indica uma relação quadrática significante entre idade (ID2) e VTP. Ou seja, a produção cresce até uma certa idade do responsável do estabelecimento, quando passa a decrescer. Além disso, o nível educacional do produtor (NE) teve forte relação positiva no VTP. Um produtor que sabia ler e escrever (NE1) apresentou um valor total médio da produção $15,7 \%$ superior quando comparado a um produtor sem instrução (referência da análise). Se o agente alcançasse o ensino fundamental completo (NE4), o VTP médio era 58,7\% superior ao encontrado para o produtor sem instrução. 0 maior impacto foi encontrado quando o agricultor tinha ensino superior (NE6) - VTP 72,9\% maior em relação ao sem instrução. O modelo indica também que, se o produtor não familiar era membro de uma cooperativa (COOP), seu VTP médio teve valor $28,4 \%$ superior frente aos não cooperados. Além disso, se dirigido por mulher, o VTP médio do estabelecimento se apresentava $25 \%$ menor frente àqueles gerenciados por homem.

Tabela 3 - Estimações do modelo, utilizando o método de 1E e 2E.

\begin{tabular}{|c|c|c|c|c|}
\hline \multirow{2}{*}{ Variável } & \multicolumn{2}{|c|}{$1 \mathrm{E}$} & \multicolumn{2}{|c|}{$2 \mathrm{E}$} \\
\hline & Coef. & & Coef. & \\
\hline Cr_Bancos & 0,2768 & *** & - & - \\
\hline Cr_Outros & 0,2862 & *** & - & - \\
\hline$\hat{Y}_{i}^{\complement} \_$Bancos & - & - & 0,4905 & *** \\
\hline$\hat{Y}_{i}^{\complement} \_$Outros & - & - & 1,1421 & $* * *$ \\
\hline$A E$ & 0,3606 & $* * *$ & 0,3590 & $* \star \star$ \\
\hline NT & 0,5393 & $* * *$ & 0,5344 & $* * *$ \\
\hline NO & 0,1929 & $* * *$ & 0,1927 & $* * *$ \\
\hline SE & 0,4755 & $* \star *$ & 0,4613 & *** \\
\hline
\end{tabular}

\footnotetext{
${ }_{4}$ Dado o limite de espaço, esses resultados não serão apresentados. No entanto, podem ser solicitados aos autores.
} 


\begin{tabular}{|c|c|c|c|c|}
\hline SU & 0,5076 & $* * *$ & 0,4788 & 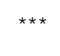 \\
\hline $\mathrm{CO}$ & 0,5184 & $* \star *$ & 0,4972 & $* \star \star *$ \\
\hline COOP & 0,2970 & $* \star * *$ & 0,2503 & $* * *$ \\
\hline $\mathrm{EC}$ & $-0,0444$ & $* \star *$ & 0,0738 & $* \star \star$ \\
\hline GEN & $-0,2938$ & $* * *$ & $-0,2925$ & $* * *$ \\
\hline ID & 0,0138 & $* * *$ & 0,0128 & $* * \star$ \\
\hline ID2 & $-0,0001$ & $* * *$ & $-0,0001$ & $* * \star$ \\
\hline NE1 & 0,1471 & $* * *$ & 0,1465 & $* * \star$ \\
\hline NE2 & 0,0995 & $* \star *$ & 0,1089 & $* \star \star$ \\
\hline NE3 & 0,2908 & $* * *$ & 0,2931 & $* \star \star *$ \\
\hline NE4 & 0,4571 & $* * *$ & 0,4620 & $* \star *$ \\
\hline NE5 & 0,5506 & $* * *$ & 0,5563 & $* * \star$ \\
\hline NE6 & 0,5338 & $* \star * *$ & 0,5479 & $* \star *$ \\
\hline PLT & 0,6858 & $* \star \star$ & 0,6510 & *** \\
\hline PLP & 0,5605 & $* * *$ & 0,5713 & $* * *$ \\
\hline PP & 0,0020 & $* * *$ & 0,0021 & $* * \star$ \\
\hline TRA & $-0,0050$ & & $-0,0061$ & \\
\hline TRM & 0,3206 & $* \star \star$ & 0,3137 & $* \star \star *$ \\
\hline OT & 0,4150 & *** & 0,4017 & $* \star \star$ \\
\hline TS & 0,3172 & 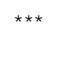 & 0,3049 & $* \star \star$ \\
\hline AGT & 0,3367 & $* * *$ & 0,3176 & $* \star *$ \\
\hline ESP_INT & 1,1320 & $* * *$ & 1,1297 & $* * *$ \\
\hline ESP_N_INT & $-0,2593$ & $* * *$ & $-0,2587$ & $* * *$ \\
\hline N_ESP_INT & 0,8536 & $* \star *$ & 0,8315 & $* \star *$ \\
\hline $\mathrm{C}$ & 4,4642 & $* * *$ & 4,6222 & $* \star \star$ \\
\hline \multicolumn{2}{|c|}{ Teste de endogeneidade $-\hat{Y}_{i}^{E}{ }_{-}$Bancos } & & $-0,3631$ & *** \\
\hline \multicolumn{2}{|c|}{ Teste de endogeneidade $-\hat{Y}_{i}^{E} \_$Ourros } & & $-0,7074$ & $* * *$ \\
\hline$R^{2}$ & 0,6018 & & 0,6010 & \\
\hline F & 11127,0 & & 33998,8 & \\
\hline
\end{tabular}

Nota: *** significância a 0,1\%; ** significância a 1\%; * significância a 5\%.

Fonte: Resultados da pesquisa.

A análise dos coeficientes relacionados às características do estabelecimento mostra que o coeficiente da variável relativa à área da propriedade $(A E)$ foi positivo e estatisticamente diferente de zero. Para um aumento em $1 \%$ na área total do estabelecimento, houve um aumento de 0,35\% no VTP. Além disso, as variáveis binárias para regiões foram positivas e estatisticamente diferentes de zero, ou seja, todas as regiões apresentaram um valor total médio da produção superior ao da região Nordeste, referência da análise.

Os coeficientes das variáveis associadas ao sistema de produção mostram que o uso da tração mecânica, acesso à orientação técnica $(O T)$, a prática de tratamentos no solo (TS) e o uso de pesticidas (AGT) tiveram um impacto positivo elevado no VTP. Um produtor não familiar com acesso à orientação 
técnica apresentou, por exemplo, um valor médio total da produção 49,4\% maior do que o de um produtor sem acesso.

Para analisar o efeito da integração de mercado e da especialização da propriedade rural no acesso a crédito, foram criadas quatro variáveis derivadas dos graus de interação e de especialização presentes no censo agropecuário. Os resultados mostram que quando os estabelecimentos se mostravam integrados ao mercado (ESP_INT e N_ESP_INT), independentemente do grau de especialização, o efeito sobre o valor da produção foi positivo e significante, em comparação aos estabelecimentos não integrados e não especializados. Para os estabelecimentos especializados, porém não integrados ao mercado (ESP_N_INT), a média do valor total da produção foi $22,7 \%$, inferior ao encontrado em estabelecimentos não especializados e não integrados.

\subsection{ESTIMATIVAS PARA AS REGIÕES BRASILEIRAS}

As estimações de $2 E$ para cada região brasileira complementam a análise da relação entre acesso aos financiamentos e valor total da produção Apêndice D. Novamente, os resultados revelam um bom ajuste do modelo às informações da amostra, como demonstram as estatísticas de qualidade de ajuste ${ }^{5}$.

O coeficiente associado à variável instrumental, $\hat{Y}_{i}^{\complement}{ }_{-}$Banco , mostra que a obtenção de financiamento via banco elevou o valor médio total da produção em todas as regiões, com destaque para o Sul, onde esse impacto foi de $245 \%$, seguidos pelo Norte $(70,5 \%)$, Sudeste $(60,4 \%)$, Centro-Oeste $(49,2 \%)$ e Nordeste (31,3\%). Esses resultados positivos encontrados vão ao encontro dos encontrados na literatura para, por exemplo, a Índia (SIDHU et al., 2008), China (XIN; LI, 2011), Paquistão (AKRAM et al., 2013) e Brasil (MOURA, 2016).

O acesso ao financiamento via outras fontes, como cooperativas de crédito, fornecedores, comerciantes, empresas integradoras, outras financeiras, ONGs, parentes e outros agentes, também apresentou resultados positivos, elevando o valor médio total da produção do Norte, Nordeste e Centro-Oeste. O resultado encontrado para o Norte e Nordeste pode ser explicado pelo fato de o grupo de acesso ao financiamento conter informações de empréstimos realizados por parentes e outros agentes, que pode suprir uma restrição ao crédito bancário para esses produtores. Para o Centro-Oeste, o impacto elevado do acesso ao financiamento, via outras fontes, pode estar associado ao fato dos grandes produtores dessa região acessarem financiamento por meio de fornecedores, por exemplo, como mencionado por Saes e Silveira (2014). Vale, contudo, lembrar que, em 2006, os estabelecimentos agropecuários que acessaram o financiamento via essa fonte representam apenas $2 \%$ da amostra.

\section{CONCLUSÕES}

O presente estudo obteve robusta evidência que o acesso ao crédito possui um impacto positivo e significativo sobre os valores de produção dos

\footnotetext{
5 Para a estimação dos modelos de 2E para cada região brasileira, foram controlados todos os fatores exógenos, incluindo os efeitos fixos para as microrregiões.
} 
agricultores brasileiros não familiares, os quais tendem a praticar a denominada agricultura empresarial.

Os dados do Censo Agropecuário de 2006 mostram que cerca de 137 mil estabelecimentos não familiares tiveram acesso a algum tipo de financiamento - considerando o total de produtores, 15,2\% utilizaram crédito bancário e $2 \%$ outras fontes. Apesar do aumento no volume de crédito direcionado ao setor rural, o número de propriedades que obteve crédito é relativamente pequeno. Apesar disso, esse grupo de produtores apresentou maior valor de produção em todas as regiões. Destaque é dado à região Centro-Oeste, onde o VTP dos estabelecimentos com acesso ao financiamento foi três vezes maior em relação aos demais. Tal resultado pode ser explicado por se tratar de uma nova fronteira agrícola no país, onde o acesso ao crédito seria o principal insumo para impulsionar a produção. Além disso, a produtividade média dos estabelecimentos com acesso ao financiamento foi superior em todas as regiões. O acesso à tecnologia e o uso de práticas mais eficientes de produção também foram mais elevados entre os produtores com acesso ao crédito, em todas as regiões.

Para analisar o impacto do acesso ao financiamento sobre o valor da produção dos produtores não familiares, utilizou-se um modelo de $2 \mathrm{E}$, uma vez que as variáveis relacionadas ao acesso ao crédito podem não ser exógenas, o que tornaria as estimativas de mínimos quadrados ordinários viesadas. Para corrigir esse problema, estimouse uma equação para crédito com a utilização da variável instrumental correspondente às dívidas totais dos estabelecimentos, sendo suas estimativas usadas na equação para o valor da produção. Os resultados demonstram que o impacto do crédito, tanto por via bancária como por outras fontes, foi positivo e significante. Variáveis relativas às características do agricultor, da propriedade e do sistema de produção também tiveram efeito significativo sobre o VTP. Estimações para cada uma das cinco regiões do País foram ainda realizadas e confirmaram, de forma geral, os resultados acima assinalados. Evidenciou-se também que o tamanho da influência de cada fonte de crédito sobre o VTP varia consideravelmente entre as regiões brasileiras.

O estudo traz uma importante contribuição para o entendimento do efeito do crédito sobre a agricultura empresarial brasileira. Ao fazer uso de microdados do IBGE, com 796.422 estabelecimentos não familiares, a pesquisa aponta para um quadro de racionamento do crédito rural no Brasil. Em especial, fornece parâmetros para formulação de políticas ao setor agropecuário ao dimensionar como o capital de terceiros influencia o valor da produção agrícola e como tal impacto se diferencia entre as diferentes fontes de crédito e entre as distintas regiões do País. Como limitação do trabalho, vale observar que se levou em conta somente o efeito do financiamento de 2006 no valor total da produção. Ou seja, não foi considerada a possível influência do crédito obtido em 2006 ou em anos anteriores sobre as características socioeconômicas e produtivas (incluindo adoção de tecnologias e práticas de gestão), o que também tende a contribuir para o aumento do valor médio da produção. 


\section{REFERÊNCIAS}

AKERLOF, G. A. The Market for "Lemons": Quality Uncertainty and the Market Mechanism. The Quarterly Journal of Economics, v. 84, n. 3, p. 488-500, 1970.

AKRAM, W.; HUSSAIN, Z.; AHMAD, N.; HUSSAIN, I. Does agriculture credit affect production efficiency? Frontier production function approach. Pakistan Economic and Social Review, v. 51, n. 2, p. 179-190, 2013. ALI, D. A.; DEININGER, K.; DUPONCHEL, M. Credit constraints and agricultural productivity: evidence from rural Rwanda. The Journal of Development Studies, v. 50, n. 5 p. 649665, 2014.

ASSUNÇÃO, J. J.; CHEIN, F. Condições de crédito no Brasil rural. Revista de Economia e Sociologia Rural, v. 45, n. 2, 2007.

BINSWANGER, H. P.; KHANDKER, S. R. The impact of formal finance on the rural economy of India. The Journal of Development Studies, v. 32, n. 2, p. 234-262, 1995.

DAMASCENO, N. P.; KHAN, A. S.; LIMA, P. V. P. S. O impacto do Pronaf sobre a sustentabilidade da agricultura familiar, geração de emprego e renda no Estado do Ceará. Revista de Economia e Sociologia Rural, v. 49, n. 1, p. 129-156, 2011.

DONG, F.; LU, J.; FEATHERSTONE, A. M. Effects of credit constraints on household productivity in rural China. Agricultural Finance Review, v. 72 n. 3 p. 402-415, 2012.
FOLTZ, J. D. Credit market access and profitability in Tunisian agriculture. Agricultural Economics, v. 30, n. 3, p. 229-240, 2004.

GOLDSMITH, R. W. Financial Structure and Development. New Haven: Yale University Press, 1969.

GUANZIROLI, C. E. PRONAF dez anos depois: resultados e perspectivas para o desenvolvimento rural. Revista de Economia e Sociologia Rural, v. 45, n. 2, p. 301-328, 2007.

GURLEY, J. G.; SHAW E. S. Financial aspects of economic development. The American Economic Review, v. 45, n. 4, p. 515-538, 1955.

GRYZAGORIDIS, O. B., FERREIRA, L.R. Impactos do Crédito Rural no Brasil. XLVI Congresso da

Instituto Brasileiro de Geografia e Estatística (2006). Censo Agropecuário 2006. Rio de Janeiro: IBGE, p. 1-777.

KABOSKI J. P, TOWNSEND R.M. The impact of credit on village economies. American Economic Journal: Applied Economics, v. 4, n. 2, p. 98133, 2012.

KHANDKER, S. R.; FARUQEE, R. R. The impact of farm credit in Pakistan. Agricultural Economics, v. 28, n. 3, p. 197-213, 2003.

KUMAR, C. S.; TURVEY, C. G.; KROPP, J. D. The impact of credit constraints on farm households: survey results from India and China. Applied Economic Perspectives Policy, v. 35, n. 3, p. 508527, 2013. 


\section{REFERÊNCIAS}

LI, R.; LI, Q.; HUANG, S.; ZHU, X. The credit rationing of Chinese rural households and its welfare loss: an investigation based on panel data. China Economic Review, v. 26, p. 1727, 2013.

LOPES, M.R.; SOUZA， G. S.; LOPES, I. V.; HONCZAR, G. O problema do acesso ao capital na agricultura brasileira. Revista de Política Agrícola, n. 4, p. 16-25, 2011.

NARAYANAN, S. The productivity of agricultural credit in India. Agricultural Economics, v. 47, p. 399-409, 2016.

MATOS, O. C. Desenvolvimento do sistema financeiro e crescimento econômico no Brasil: evidências de causalidade. Working Paper $\mathrm{n}^{\circ}$ 49. Banco Central do Brasil, 2002.

MOURA, F. R. O nexo causal entre crédito rural e crescimento do produto agropecuário na economia brasileira. Tese - Escola Superior de Agricultura "Luiz de Queiroz", Universidade de São Paulo, 2016.

PINDYCK, R. S.; RUBINFEL, D. L. Econometria: modelos e previsões. Rio de Janeiro: Elsevier, 2004.

ROBINSON J. The Generalization of the General Theory. In: The Rate of Interest and Other Essays. London: MacMillan. 1952.
ROOYEN, C. V.; STEWART, R.; WET, T. The impact of microfinance in Sub-Saharan Africa: a systematic review of the evidence. World Development, $v$. 40, p. 2249-2262, 2012.

SAES, M. S. M.; SILVEIRA, R. L. F. S. Novas formas de organização das cadeias agrícolas brasileiras: tendências recentes. Estudos Sociedade e Agricultura, v. 22, p. 386-407, 2014.

SCHUMPETER, J. A. The theory of economic development. Cambridge, MA: Harvard University Press, 1911.

SIDHU, R. S.; VATTA, K.; KAUR, A. Dynamics of institutional agricultural credit and growth in Punjab: contribution and demand-supply gap. Agricultural Economics Research Review, v. 21, p. 407-414, 2008.

SPOLADOR, H. F. S. Reflexões sobre a experiência brasileira de financiamento agrícola. Piracicaba: Universidade de São Paulo, 93p. Dissertação, 2001.

STIGLITZ, J., WEISS, A. Credit rationing in markets with imperfect information. American Economic Review, v. 71, n. 3, p. 333-421, 1981.

XIN, L.; LI, Y. Relationship between agricultural credits and agricultural economy based on error correct model in Heilongjiang province. Journal of Northeast Agricultural University, v. 18, n. 1, p. 75-78, 2011. 


\section{REFERÊNCIAS}

TU, T. T. T.; HA, N. P.; YEN, T. T. H. Socioeconomic impact of rural credit in northern Vietnam: does it differ between clients belonging to the ethnic majority and the minorities? Asian Social Science, v. 11, p. 159-167, 2015.

YARON, J., BENJAMIN, M., Piprek, G. Rural finance: issues, design and best practice. Washington: World Bank, 1997.

ZHU, X.; Li, Z. Heterogeneous impact of farmer credit: an empirical investigation based on IVQR model. Systems Engineering - Theory \& Practice, v. 27, n. 2, p. 68-75, 2007.

\section{Gabriela dos Santos Eusébio'}

Graduada em Economia pela Faculdade de Administração, Economia e Contabilidade de Ribeirão Preto (FEA-RP), Universidade de São Paulo (2008), mestre em Economia Aplicada pela Faculdade de Administração, Economia e Contabilidade de Ribeirão Preto (FEA-RP), Universidade de São Paulo (2011) e doutora em Ciências Econômicas pelo Instituto de Economia da Universidade Estadual de Campinas, Unicamp (2017). Núcleo de Economia Agrícola e do Meio Ambiente (NEA) da Universidade Estadual de Campinas - Unicamp. E-mail: gabeusebio@gmail.com

\section{Alexandre Gori Maia²}

Graduado em Estatística pela Universidade Estadual de Campinas (1996), mestre em Desenvolvimento Econômico pela Universidade Estadual de Campinas (2001) e doutor em Economia Aplicada pela Universidade Estadual de Campinas (2006). É Professor Livre Docente da Universidade Estadual de Campinas, no Instituto de Economia. Tem experiência em econometria e desenvolvimento econômico, atuando principalmente nas áreas: estratégias de desenvolvimento sustentável, impactos da migração rural no desenvolvimento econômico, desenvolvimento da estrutura social e desigualdade. Núcleo de Economia Agrícola e do Meio Ambiente (NEA) da Universidade Estadual de Campinas Unicamp. E-mail: gori@unicamp.br

\section{Rodrigo Lanna Franco da Silveira ${ }^{3}$}

Graduado em Economia pela Faculdade de Administração, Economia e Contabilidade da Universidade de São Paulo - FEA/USP (1999), mestre e doutor em Economia Aplicada pela Esalq/USP (2009). É Professor Livre Docente da Universidade Estadual de Campinas, no Instituto de Economia. A sua pesquisa se concentra em economia agrícola, economia de empresas e finanças. Núcleo de Economia Agrícola e do Meio Ambiente (NEA) da Universidade Estadual de Campinas - Unicamp. E-mail: rlanna@unicamp.br

Datas de Submissão: 17/09/2018

Data de Aceite: 17/12/2019 


\section{APÊNDICES}

Apêndice A - Lista de variáveis do estabelecimento agropecuário e do produtor.

\begin{tabular}{|c|c|}
\hline Variável & Descrição \\
\hline IDADE_DIR - ID & Idade em anos da pessoa que dirige o estabelecimento \\
\hline FEMININO- GEN & variável binária que assume o valor 1 se o diretor do estabelecimento for mulher e 0 caso contrário \\
\hline ESCOLA01- NE1 & variável binária de instrução; 1 se escolaridade máxima é saber ler e escrever e 0 caso contrário \\
\hline ESCOLA02- NE2 & variável binária de instrução; 1 se escolaridade máxima é alfabetização de adultos e 0 caso contrário \\
\hline ESCOLA10- NE3 & variável binária de instrução; 1 se escolaridade máxima é o fundamental incompleto e 0 caso contrário \\
\hline ESCOLA11- NE4 & variável binária de instrução; 1 se escolaridade máxima é o fundamental completo e 0 caso contrário \\
\hline ESCOLA21- NE5 & variável binária de instrução; 1 se escolaridade máxima é o ensino médio completo e 0 caso contrário \\
\hline ESCOLA31- NE6 & variável binária para instrução; 1 se escolaridade máxima é o superior completo e 0 caso contrário \\
\hline PART_COOP-COOP & variável binária que assume o valor 1 se o produtor é cooperado e 0 caso contrário \\
\hline PART_ENTID- EC & variável binária que assume o valor 1 se o produtor é associado à entidade de classe e 0 caso contrário \\
\hline LAVOURA- AL & área total de lavouras em hectares \\
\hline PASTAGEM- AP & área total de pastagens em hectares \\
\hline AF_PRES- AFP & área de florestas naturais preservadas em hectares \\
\hline AF_NAT_EXP-AFE & área de florestas naturais exploradas em hectares \\
\hline AF_AGROF- ASA & área com sistemas agroflorestais em hectares \\
\hline OUTROS- AOU & área com outra utilização em hectares (florestas plantadas, lagos, construção, etc.) \\
\hline PVAPANIMAL- PVPA & valor da produção animal sobre valor total da produção \\
\hline PVPVEGETAL- PVPV & valor da produção vegetal sobre valor total da produção \\
\hline PVPAGRO- PVAA & valor agregado da agroindústria sobre valor total da produção \\
\hline PERC_LAVOURAS- PL & percentual de áreas de lavouras \\
\hline PLAV_TEMP- PLT & percentual de áreas de lavouras temporárias \\
\hline PLAV_PERM- PLP & percentual de áreas de lavouras permanentes \\
\hline PERC_PASTAGENS- PP & percentual de áreas de pastagens \\
\hline PERC_MATAS- PM & percentual de áreas de matas \\
\hline CR_BANCOS & variável binária com valor 1 se o estabelecimento acessou crédito bancário e 0 caso contrário \\
\hline CR_COOP & variável binária com valor 1 se o estabelecimento acessou crédito via cooperativas de crédito e 0 caso contrário \\
\hline CR_OUTROS & variável binária com valor 1 se o estabelecimento acessou crédito via outras fontes de crédito e 0 caso contrário \\
\hline AREA_TOTAL- AE & área do estabelecimento em hectares \\
\hline VT_PRODUCAO- VTP & valor total da produção em $\mathrm{R} \$$ \\
\hline DIVTOT-DT & valor total das dívidas do estabelecimento \\
\hline NO & variável binária com valor 1 se estabelecimento pertence à região Norte e 0 caso contrário \\
\hline NE & variável binária com valor 1 se estabelecimento pertence à região Nordeste e 0 caso contrário \\
\hline SE & variável binária com valor 1 se estabelecimento pertence à região Sudeste e 0 caso contrário \\
\hline SU & variável binária com valor 1 se estabelecimento pertence à região Sul e 0 caso contrário \\
\hline $\mathrm{CO}$ & variável binária com valor 1 se estabelecimento pertence à região Centro-Oeste e 0 caso contrário \\
\hline
\end{tabular}


Apêndice B - Lista de variáveis das características do sistema de produção.

\begin{tabular}{|c|c|}
\hline Variável & Descrição \\
\hline TRACAO- TRAM & $\begin{array}{l}\text { variável binária com valor } 1 \text { se o produtor utiliza força de tração animal e/ou mecânica e } 0 \text { caso } \\
\text { contrário }\end{array}$ \\
\hline TRACAO_ANIMAL-TRA & variável binária com valor 1 se o produtor utiliza força de tração animal e 0 caso contrário \\
\hline TRACAO_MECANICA-TRM & variável binária com valor 1 se o produtor utiliza força de tração mecânica e 0 caso contrário \\
\hline ORIENTACAO- OT & variável binária com valor 1 se o produtor recebe orientação técnica e 0 caso contrário \\
\hline TRATAMENTOSOLO- TS & $\begin{array}{l}\text { variável binária com valor } 1 \text { se o produtor faz aplicação de corretivo do ph do solo ou adubação e } 0 \\
\text { caso contrário }\end{array}$ \\
\hline PESTICIDA- PEST & $\begin{array}{l}\text { variável binária com valor } 1 \text { se o produtor utiliza pesticida para controle de pragas e/ou doenças e } 0 \\
\text { caso contrário }\end{array}$ \\
\hline ESPECIALIZADO1-ESSE & variável binária com valor 1 se o estabelecimento é superespecializado e 0 caso contrário \\
\hline ESPECIALIZADO2- ESP & variável binária com valor 1 se o estabelecimento é especializado e 0 caso contrário \\
\hline ESPECIALIZADO3-EDIV & variável binária com valor 1 se o estabelecimento é diversificado e 0 caso contrário \\
\hline ESPECIALIZADO4-EMDIV & variável binária com valor 1 se o estabelecimento é muito diversificado e 0 caso contrário \\
\hline INTEGRADO1-EMI & variável binária com valor 1 se o estabelecimento é muito integrado e 0 caso contrário \\
\hline INTEGRADO2-EI & variável binária com valor 1 se o estabelecimento é integrado e 0 caso contrário \\
\hline INTEGRADO3-EPI & variável binária com valor 1 se o estabelecimento é pouco integrado e 0 caso contrário \\
\hline ESP_INT & variável binária com valor 1 se o estabelecimento é especializado e integrado, e 0 caso contrário \\
\hline N_ESP_INT & variável binária com valor 1 se o estabelecimento é não especializado e integrado, e 0 caso contrário \\
\hline ESP_N_INT & variável binária com valor 1 se o estabelecimento é especializado e não integrado, e 0 caso contrário \\
\hline N_ESP_N_INT & $\begin{array}{l}\text { variável binária com valor } 1 \text { se o estabelecimento é não especializado e não integrado, e } 0 \text { caso } \\
\text { contrário }\end{array}$ \\
\hline MDO_TOTAL-NT & Somatório do trabalho contratado e do trabalho familiar. \\
\hline
\end{tabular}


Apêndice C - Estimativas de máxima verossimilhança da regressão logística multinomial.

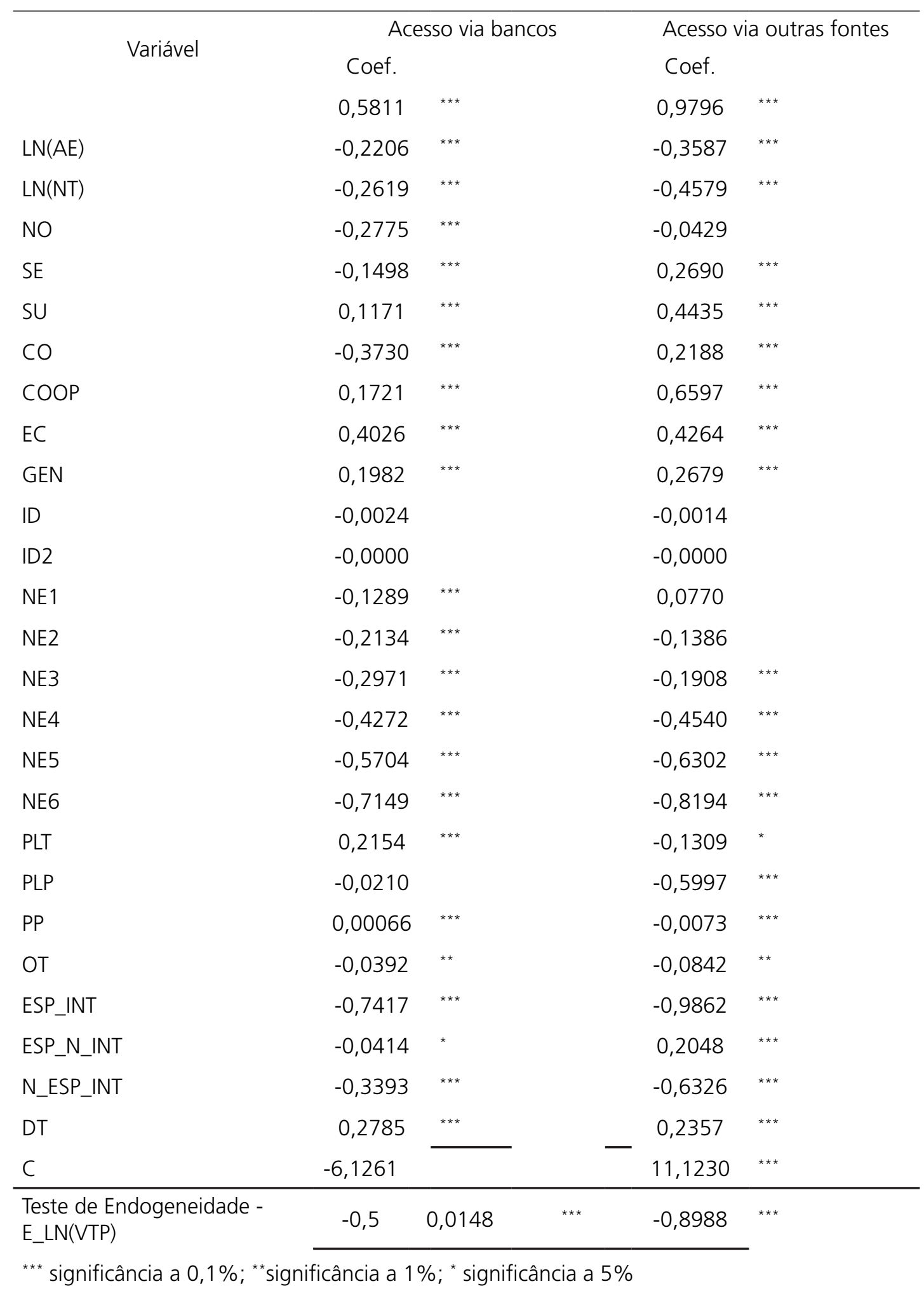


Apêndice D - Estimações do modelo, em MQ2E, por região do Brasil.

\begin{tabular}{|c|c|c|c|c|c|c|c|c|c|c|}
\hline 1Grí́ & Norte & & Nordeste & & Sudeste & & Sul & & Centro-Oest & \\
\hline variave? & Coef. & & Coef. & & Coef. & & Coef. & & Coef. & \\
\hline$Y_{i}^{C r}$ Bancos & 0,534 & $* \star \star *$ & 0,2726 & $* \star \star$ & 0,4726 & 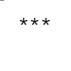 & 1,2412 & $* * *$ & 0,4003 & *** \\
\hline$Y_{i}^{C r}$ Coutros & 4,0374 & $* * *$ & 5,4896 & $* \star \star$ & $-0,4298$ & ** & $-3,7177$ & $* * *$ & 0,9674 & *** \\
\hline $\mathrm{AE}$ & 0,2646 & $* \star \star$ & 0,3403 & $* \star \star$ & 0,3799 & $* * *$ & 0,3443 & *** & 0,3838 & *** \\
\hline NT & 0,4828 & $* * *$ & 0,4973 & $* * *$ & 0,5026 & $* * *$ & 0,5924 & $\star * \star *$ & 0,4813 & $* * *$ \\
\hline COOP & 0,1727 & $* * *$ & 0,2325 & $* * *$ & 0,2928 & $* * *$ & 0,3748 & *** & 0,1668 & *** \\
\hline EC & $-0,0259$ & & $-0,1255$ & $* \star \star$ & $-0,0037$ & & $-0,0318$ & 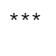 & $-0,0112$ & \\
\hline GEN & $-0,2442$ & $* * *$ & $-0,3631$ & $* * *$ & $-0,2400$ & $* * \star$ & $-0,2806$ & *** & $-0,1704$ & $* * *$ \\
\hline ID & 0,0129 & $* * *$ & 0,0167 & $* * \star$ & 0,0055 & $* * *$ & 0,0124 & *** & 0,0019 & \\
\hline ID2 & $-0,0001$ & $* * *$ & $-0,0001$ & $* * *$ & $-0,0001$ & $* \star \star$ & $-0,0001$ & $* \star \star$ & $-0,0001$ & \\
\hline NE1 & 0,0716 & * & 0,1292 & $* \star \star$ & 0,2540 & $* * *$ & 0,2398 & *** & 0,1643 & $* * *$ \\
\hline NE2 & 0,0378 & & 0,0543 & $* *$ & 0,2766 & $* * *$ & 0,0946 & ** & 0,0871 & * \\
\hline NE3 & 0,1934 & $* * \star$ & 0,2691 & 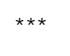 & 0,4267 & $* * *$ & 0,3698 & $* * *$ & 0,1977 & $* \star \star$ \\
\hline NE4 & 0,3291 & $* \star \star$ & 0,4699 & $* * \star$ & 0,5375 & ${ }^{* * *}$ & 0,5125 & 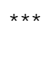 & 0,3394 & $* * \star$ \\
\hline NE5 & 0,4247 & $* * \star$ & 0,5687 & $* * *$ & 0,6050 & $* * *$ & 0,6312 & 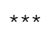 & 0,4482 & *** \\
\hline NE6 & 0,3624 & $* * *$ & 0,5979 & $* * *$ & 0,5799 & $* * *$ & 0,6047 & $* * *$ & 0,4783 & $* * *$ \\
\hline PLT & 0,8792 & $* \star \star$ & 0,3596 & $* \star \star$ & 0,9190 & $* * \star$ & 0,4471 & $* * *$ & 1,7103 & $* * *$ \\
\hline PLP & 0,3881 & $* * *$ & 0,5088 & $* * *$ & 0,8067 & $* \star \star$ & $-0,1967$ & *** & 0,4774 & $* \star \star$ \\
\hline PP & 0,0050 & $* * *$ & 0,0026 & 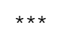 & 0,0012 & *** & $-0,0004$ & * & 0,0038 & $* \star \star$ \\
\hline TRA & 0,1769 & $* * \star$ & 0,1217 & $* * *$ & $-0,0337$ & $* * \star$ & $-0,0341$ & $* * \star$ & $-0,0093$ & \\
\hline TRM & 0,4883 & $* * *$ & 0,2353 & $* * \star$ & 0,3593 & $* * *$ & 0,3609 & *** & 0,3676 & $* * *$ \\
\hline OT & 0,2087 & $* \star \star *$ & 0,4247 & $* * \star$ & 0,3250 & $* * *$ & 0,5327 & $* * *$ & 0,2951 & $* * *$ \\
\hline TS & 0,1153 & $* \star \star$ & 0,4073 & 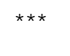 & 0,2656 & $* \star \star$ & 0,1654 & 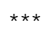 & 0,2284 & $* * *$ \\
\hline PEST & 0,3171 & $* \star \star$ & 0,2978 & 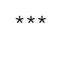 & 0,3230 & $* * *$ & 0,2405 & $* * *$ & 0,2367 & $* * *$ \\
\hline ESP_INT & 1,2463 & $* \star \star$ & 0,9435 & *** & 1,2255 & $* \star \star$ & 0,9777 & *** & 1,6405 & $* * *$ \\
\hline $\begin{array}{l}\text { ESP_N_ } \\
\text { INT }\end{array}$ & $-0,5898$ & $* \star \star$ & $-0,1934$ & 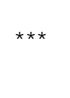 & $-0,1585$ & *** & 0,0081 & & $-0,5653$ & $* * *$ \\
\hline $\begin{array}{l}N_{-} E S P_{-} \\
\text {INT }\end{array}$ & 0,8427 & $* * *$ & 0,6143 & $* * \star$ & 1,0170 & $* * *$ & 0,7612 & *** & 1,4126 & $* * *$ \\
\hline C & 5,0749 & $* * \star$ & 4,7453 & $* * *$ & 5,0703 & $* * *$ & 5,1622 & *** & 4,8280 & $* * *$ \\
\hline$R^{2}$ & 0,4581 & & 0,5038 & & 0,5511 & & 0,5874 & & 0,6013 & \\
\hline $\mathrm{F}$ & 943,13 & & 4067,8 & & 2564,81 & & 3094,48 & & 2737,33 & \\
\hline$N$ & 52.482 & & 232.388 & & 190.146 & & 139.134 & & 85.351 & \\
\hline
\end{tabular}

*** significância a 0,1\%; * significância a 1\%; ${ }^{*}$ significância a 5\% 